\title{
Triagem do processamento auditivo em escolares de 8 a 10 anos
}

\section{Triagem do processamento auditivo}

\author{
Larissa Fortunato Simon \\ Angela Garcia Rossi
}

\section{Resumo}

É fundamental que existam instrumentos válidos e confiáveis para detectar as dificuldades do processamento auditivo em crianças em idade escolar muitas vezes associadas aos transtornos de aprendizagem. Esta pesquisa teve como objetivo avaliar o desempenho de uma triagem do processamento auditivo em escolares para diferenciar grupos em função da idade. Os resultados revelaram um aumento no desempenho médio nas provas em função da idade.

Palavras-chaves: Triagem; Processamento auditivo; Escolares.

\section{Auditory processing screening in school children from 8 to 10 years old}

\begin{abstract}
It's essential the existence of valid and precise instruments to detect auditory processing difficulties in school-aged children, which are frequently associated to learning disabilities. This research aimed to analyze an auditory processing screening in school-aged children to discriminate groups acording to the age. The results related a development in the average performance acording to the age.

Key-words: Screening; Auditory Processing; School-aged children.
\end{abstract}

\section{Selección del Procesamiento auditivo en escolares de 8 a 10 años}

\section{Resumen}

Es fundamental que existan instrumentos válidos y confiables para detectar las dificultades de procesamiento auditivo en niños con edad escolar, muchas veces asociadas a los trastornos de aprendizaje. Esta investigación tuvo como objetivo evaluar el desempeño de una selección del procesamiento auditivo en escolares con relación a su capacidad de diferenciar grupos en función de la edad. Los resultados mostraron mejoría de los promedios de aciertos conforme el aumento de la edad.

Palabras-clave: Selección; Procesamiento auditivo; Escolares. 


\section{Introdução}

Os transtornos de aprendizagem têm sido foco de inúmeras pesquisas durante os últimos anos. Tal interesse por parte de diversas áreas da ciência devese à importância da aprendizagem para o completo desenvolvimento sócio-cultural e até mesmo emocional do ser humano.

A Fonoaudiologia apresenta um papel essencial na avaliação e tratamento desses transtornos, já que é a ciência que estuda a comunicação humana e seus distúrbios, englobando as áreas de audição, linguagem, voz e motricidade oral. Sua contribuição nas pesquisas com respeito à aprendizagem tem sido fundamental para o desenvolvimento de novas teorias e condutas, possibilitando um desenvolvimento ímpar nessa área de conhecimento.

A abordagem que relaciona os transtornos da aprendizagem e os déficits no processamento da informação auditiva é considerada de inestimável relevância na comunidade científica, não só na área dos Distúrbios da Comunicação Humana, mas também nas Neurociências e Neuroaudiologia.

O Processamento Auditivo (PA) refere-se aos mecanismos e processos do sistema auditivo responsáveis pelos fenômenos comportamentais de localização e lateralização sonora; discriminação auditiva; reconhecimento de padrões auditivos; aspectos temporais da audição, incluindo resolução, mascaramento, integração e ordenação temporal; e desempenho auditivo na presença de sinais acústicos degradados ou competitivos (American SpeechLanguage-Hearing Association - ASHA, 1996).

Segundo Keith e Pensak (I99I), a Desordem do Processamento Auditivo (DPA) é a inabilidade do indivíduo em atender, discriminar, reconhecer, recordar e/ou compreender informações apresentadas aos canais auditivos, mesmo apresentando níveis normais de inteligência e audição periférica.

Para Katz e Wilde (1989), PA é a construção que se faz acerca do sinal auditivo para tornar a informação funcionalmente útil. Eles também relatam que os transtornos de aprendizagem, especialmente os problemas de leitura e as dificuldades com fonemas, com a compreensão leitora e os comprometimentos de ortografia e de habilidades com língua estrangeira estão associados às DPAs.

Segundo Keith (1988), as principais características das DPAs são as alterações na atenção dirigida, fadiga em tarefas complexas ou prolongadas, distração, sensibilidade exagerada frente a sons intensos, dificuldades em seguir ordens verbais, necessidade de repetição freqüente de estímulos verbais, alterações mnésicas, aprendizado lento das relações grafofonêmicas além de dificuldades em compreender piadas e linguagem figurada. Para Chermak e Musiek (1992), os déficits de compreensão da fala na presença de ruído, a dificuldade de comunicação e o baixo desempenho acadêmico seriam incompatíveis com a inteligência. Já Bellis (1996) e Ferre (1997) afirmaram que as crianças com DPA apresentam dificuldade em identificar a idéia principal de um enunciado, baixa capacidade de interpretação de palavras, frases, anedotas, metáforas, trocadilhos e analogias de sentido ambíguo, alteração na emissão verbal e dificuldade de resgate verbal.

As causas das DPAs podem ser as mais diversas, destacando-se as otites freqüentes na primeira infância, febres altas e contínuas, distúrbios específicos no desenvolvimento da função auditiva, disfunções subclínicas, ou mesmo pequenas lesões em alguma etapa das vias auditivas e/ou privação sensorial auditiva durante a primeira infância (Alvarez, Caetano \& Nastas, 1997). Baran e Musiek (1999) citam os atrasos no desenvolvimento neuromaturacional do Sistema Nervoso Auditivo Central (SNAC), alterações nesse sistema devido a lesões similares àquelas encontradas em adultos ou a presença de déficits perceptuais auditivos e/ou lingüísticos.

Os estudos de processamento auditivo tiveram início na tentativa de explicar as queixas auditivas de adultos com lesões no SNAC, mas que tinham acuidade auditiva normal. Descobriu-se, porém, que a avaliação central da audição também é muito útil e sensível para avaliar a integridade funcional do SNAC, dando aos clínicos mais subsídios no diagnóstico diferencial como também intervenção das dificuldades de linguagem e transtornos de aprendizagem.

Muitos autores relacionam os transtornos de aprendizagem e as DPAs. Utilizando diferentes testes 
comportamentais e faixas de idade com diversas metodologias eles mostram que grande parte das crianças que apresenta dificuldades acadêmicas tem escores abaixo do esperado nos testes que avaliam a função auditiva (Flowers, 1964; Willeford, 1976; White, 1977; Katz, 1992; Ribas-Guimarães, 2000; Sanches \& Alvarez, 2000; Costamilan, 200I; Costa, 2003; Costamilan, 2004; Damasceno \& Russo, 2004; Frota, 2004). Por isso é fundamental que os profissionais envolvidos no acompanhamento de crianças com transtornos de aprendizagem tenham consciência da importância da avaliação do processamento auditivo nessa população.

Capovilla, Capovilla e Silveira (2000) comentaram que diversas teorias buscam explicar as causas das dificuldades de leitura. Entre elas estão os distúrbios no processamento seqüencial de estímulos apresentados rapidamente ou com curtos intervalos, incluindo estímulos auditivos verbais e não-verbais, percepção de fala, processamento da informação com demanda de memória de curto prazo e longo prazo.

A ênfase atual na avaliação auditiva central em crianças com distúrbio de aprendizagem, segundo Musiek e Lamb (1999), surgiu da necessidade de identificar prejuízos auditivos sutis, que poderiam interferir no trabalho acadêmico e nas habilidades sociais e/ou de comunicação.

No Brasil pode-se contar com diferentes tipos de avaliações comportamentais que diagnosticam as habilidades auditivas. Mas, apesar do variado número de testes diagnósticos, não há nenhum programa sistemático de triagem da função auditiva em escolares, disponível no mercado brasileiro. As conseqüências da inexistência desse tipo de instrumento são a falta de divulgação no meio escolar desse tipo de abordagem, o grande número de diagnósticos equivocados em crianças com dificuldades acadêmicas, a impossibilidade de realizar um levantamento epidemiológico mais amplo da população escolar com relação ao Processamento Auditivo, entre outros. Além disso, os profissionais envolvidos com a aprendizagem escolar como Professores, Pedagogos, Fonoaudiólogos e Psicólogos, ao terem acesso a um instrumento válido e eficaz na identificação dos indivíduos, podem arriscar desenvolvimento de Desordens de Processamento Auditivo. Sendo assim, estes poderiam fazer encaminhamentos precoces, a fim de minimizar as dificuldades enfrentadas pelos escolares, por meio de uma terapêutica mais específica e eficaz, evitando e/ ou diminuindo a repetência e a evasão escolar.

Northen e Downs (1989) preconizaram que a triagem é o processo de aplicação de medidas rápidas e simples em um grande número de indivíduos, nos quais serão identificados, com alto grau de confiabilidade e validade, as alterações da função testada. Para Bellis e Burke (1996), o objetivo da triagem é oferecer informações preliminares sobre as características do indivíduo, principalmente aquelas que podem interferir em sua saúde, educação ou bem estar. Já Bess e Humes (1998) acreditam que a triagem deve ser um procedimento válido, fidedigno, uniforme e padronizado bem aceito pelos avaliandos, de aplicação rápida e fácil e em ambiente natural.

Com o intuito de detectar as possíveis causas do baixo desempenho acadêmico de crianças em idade escolar de maneira rápida, uniforme e padronizada, Keith (1986) desenvolveu uma bateria de triagem comportamental do processamento auditivo denominada SCAN (Screening Test for Central Auditory Processing Disorders). Essa bateria foi idealizada para ser administrada em sala silenciosa utilizando apenas um aparelho de som estereofônico e dois conjuntos de fones de ouvido. A duração da bateria é de aproximadamente 20 minutos, sendo composta de 3 subtestes: Palavras filtradas (Filtered Words - FW); Figura-fundo auditiva (Auditory Figure-ground - AFG) e Palavras competitivas (Competing Words - CW).

Com a utilização de um questionário, Emanuel (2002) investigou quais eram as práticas mais comuns em se tratando de avaliação do Processamento Auditivo na população pré-escolar até 18 anos por parte dos audiologistas americanos. Concluiu que juntamente com o SSW (Staggered Spondaic Word Test - Katz, 1992), os testes SCAN, SCAN-C e SCAN-A de Keith (versões atualizadas do original de 1986), foram os mais utilizados pelos audiologistas americanos.

Considerando a necessidade de instrumentos de triagem/avaliação que contemplem as características fonético-fonológicas, prosódicas e semânticas da 
Língua Portuguesa, bem como as características da população a que se destina, Zaidan (200I) elaborou uma Bateria de Triagem do Processamento Auditivo para pré-escolares e escolares, baseada no SCAN (Keith, 1986). Os testes foram desenvolvidos a partir de listas de palavras dissilábicas paroxítonas do português brasileiro retiradas de livros didáticos utilizados por crianças entre 6 e II anos. Essas listas foram submetidas a um balanceamento fonético que consistiu na comparação do corpus de referência (formado pelo levantamento da freqüência de ocorrência dos segmentos fônicos dos verbetes contidos no Minidicionário Aurélio) e no corpus das listas. As listas alcançaram grau de correlação maior de 0,95, segundo o Teste de Correlação de Sperman, sendo assim consideradas adequadas para aplicação. O material foi gravado em CD (Compact Disc) por um locutor profissional, com tempo total de 15 minutos e idealizado para ser apresentado sem a utilização de um audiômetro, o que facilita sua administração no próprio ambiente escolar. A bateria é composta por três subtestes: Fala Filtrada (FF), Fala no Ruído (FR) e Palavras Competitivas (PC).

Zaidan (200I) avaliou 60 crianças com desenvolvimento normal e 10 crianças com diagnóstico de Desordem do Processamento Auditivo na Bateria de Triagem do Processamento Auditivo. Os resultados das crianças normais mostraram uma melhora nos escores de acertos dos indivíduos conforme $o$ aumento da idade, efeito estatisticamente significativo para o escore obtido pelos participantes e também para cada subteste isoladamente, com exceção do subteste Fala no Ruído. Com relação à comparação dos escores de acertos das crianças com diagnóstico de DPA e daquelas consideradas normais, as crianças com diagnóstico de DPAs, de maneira geral, obtiveram um desempenho inferior. Sendo assim, Zaidan (200I) constatou que a Bateria de Triagem do Processamento Auditivo foi testada, aperfeiçoada e considerada adequada e sensível para avaliar escolares entre 6 e II anos de idade, apesar de ser necessário mais estudos com diferentes populações e objetivos.

A fim de esclarecer alguns pontos aparentemente obscuros no trabalho de Zaidan (200I), Capovilla
(2004) realizou uma análise dos dados brutos das avaliações da autora e aplicou testes estatísticos mais condizentes com a realidade da amostra. Ele utilizou - Teste Ancovas, seguido de análises de comparação de pares, controlando o efeito da idade como covariante. A partir dessas análises verificou que a bateria de Zaidan (200I) é válida e eficaz, tanto para analisar o efeito da faixa etária quanto para diferenciar as crianças normais daquelas que apresentam DPA. O autor ressaltou a qualidade do teste e o cuidado que a autora teve ao fornecer os dados brutos de sua pesquisa, sem os quais não haveria possibilidade de realizar essas análises.

Esta pesquisa teve como objetivo avaliar o desempenho de uma nova proposta da Bateria de Triagem do Processamento Auditivo em Escolares. A variável analisada foi a idade para grupos de 8, 9 e 10 anos nos subtestes Fala Filtrada, Fala no Ruído, Palavras Competitivas e Total da Bateria. Essa variável foi escolhida, pois a capacidade de diferenciar grupos etários, ou seja, a melhora dos resultados conforme o aumento da idade, é desejável num teste de Processamento Auditivo, já que corrobora com as evidências de desenvolvimento das habilidades auditivas e a maturação do SNAC em crianças. Como o teste se encontra em fase experimental, considerouse mais adequado realizar uma análise prévia em crianças sem evidências significativas de alterações nas áreas investigadas.

\section{Método}

\section{Participantes}

Foram avaliados 106 alunos, entre 8 e 10 anos, regularmente matriculados na segunda e terceira séries do ensino fundamental de uma escola da rede pública estadual e de uma escola da rede particular de ensino ambas num município gaúcho. Os critérios de inclusão como participante do presente estudo foram: não ter repetência escolar; evidências de quaisquer desordens significativas de comunicação, aprendizagem, desenvolvimento neuropsicomotor, linguagem ou audição; não possuir histórico de otites de repetição (mais de dois episódios por ano) ou apresentar 
quaisquer alterações à meatoscopia e à triagem audiométrica. Além desses, foi necessário revelarem compreensão e atenção suficientes para realização das atividades propostas. Analisando as avaliações prévias, 57 indivíduos preencheram os critérios de inclusão na amostra, sendo 33 do gênero feminino e 24 do gênero masculino.

\section{Avaliações}

Meatoscopia

Visualização do conduto auditivo externo a fim de excluir quaisquer impedimentos na condução aérea do som, como por exemplo, excesso de cerúmen, presença de corpo estranho, perfuração timpânica, entre outros. $O$ procedimento foi realizado utilizando um otoscópio marca Riester.

\section{Triagem audiométrica}

Verificação audiométrica através de tons puros nas freqüências de 500, 1000, 2000, 3000, 4000, 6000 e $8000 \mathrm{~Hz}$, utilizando como critério de corte a intensidade de $20 \mathrm{~dB}$ para todas as freqüências em ambas orelhas. Assim, passaram na triagem audiométrica as crianças que responderam a todos os estímulos a $20 \mathrm{~dB}$ de intensidade e falharam as que não conseguiram responder a pelo menos um dos estímulos. Esse procedimento foi realizado a fim de assegurar níveis mínimos de audição periférica, para não haver contaminação de possíveis perdas auditivas nos resultados dos testes. $O$ procedimento foi realizado utilizando um audiômetro, marca Interacoustics, modelo AS7 e fones marca Telephonics, modelo TDH 39P previamente aferidos e calibrados.

Bateria de Triagem Auditiva (Zaidan, 200I)

O subteste Fala Filtrada (FF) consiste na apresentação de 44 palavras submetidas à filtragem passa-baixo, com freqüência de corte de $1000 \mathrm{~Hz}$, com banda de rejeição de $18 \mathrm{~dB}$ por oitava.

O subteste Fala no Ruído (FR) é composto por 44 palavras apresentadas concomitantemente a um ruído competitivo ipsilateral (palavras e ruído na mesma orelha) do tipo rumor de conversação (bable), na relação sinal/ruído de $+5 \mathrm{~dB}$, ou seja, o sinal de fala se encontra com $5 \mathrm{~dB}$ mais intenso do que o ruído.
O subteste Palavras Competitivas (PC) trata de 108 palavras, apresentadas aos pares, uma à $O D$ e outra à $\mathrm{OE}$, ao mesmo tempo (estimulação dicótica).

O procedimento de aplicação dessa bateria foi realizado utilizando um aparelho de som estéreo portátil - Discman, marca Sony, modelo D-I7I; um CD (Compact Disc), contendo a Bateria de Triagem do Processamento Auditivo de Zaidan (200I), fornecido pela autora, e dois conjuntos de fones de ouvido estéreo, marca Philips, modelo SBC HPI95, um para a criança avaliada (permitindo total vedação do pavilhão auricular) e outro para avaliadora a fim de monitorar o andamento dos testes. $O C D$ apresenta os três subtestes da bateria: Fala Filtrada, Fala no Ruído e Palavras Competitivas, nesta ordem de apresentação, sempre precedidos da instrução de cada tarefa. Os subtestes: Fala Filtrada e Fala no Ruído iniciam com duas palavras-treino à orelha direita sucedidas por duas palavras treino à orelha esquerda, depois vinte palavras-teste à orelha direita e finalmente vinte palavras-teste à orelha esquerda. O subteste Palavras Competitivas inicia com quatro pares de palavras-treino, sucedidos de cinqüenta pares de palavras-teste, dados à orelha direita e orelha esquerda ao mesmo tempo (tarefa dicótica). O volume do Discman foi estabelecido através do nível de conforto da própria avaliadora no ambiente de testagem e permaneceu fixo em todas as avaliações. Cada criança foi orientada a prestar atenção no que ouvia através dos fones, já que as instruções dos testes estavam contidas no material gravado. Tendo em mãos o protocolo de avaliação (em Anexo I), os acertos são contabilizados a partir no número de palavras repetidas corretamente em cada subteste. Qualquer omissão e/ou substituição de natureza fonético-fonológica foi considerado como erro.

\section{Procedimento}

Esta pesquisa encontra-se devidamente registrada no Gabinete de Projetos do CCS/UFSM, tendo sido aprovada pelo Comitê de Ética do CCS/UFSM. Seguindo as recomendações éticas nas pesquisas que envolvem seres humanos, só foram avaliadas as crianças cujos pais aceitaram participar da pesquisa 
com livre arbítrio e sem coação através da assinatura do Termo de Consentimento Livre e Esclarecido.

Primeiramente foi estabelecido um contato com a coordenação pedagógica das escolas, a fim de apresentar o projeto, salientar sua importância e propor um cronograma de trabalho. Após a aceitação e a autorização das escolas, uma reunião com os pais e professores nas dependências das escolas foi realizada, para apresentar a proposta, demonstrar riscos e benefícios, bem como sanar possíveis dúvidas. Os pais que aceitaram participar da pesquisa assinaram o Termo de Consentimento Livre e Esclarecido e preencheram o Questionário aos Pais, que continha questões de múltipla escolha sobre o desenvolvimento neuropsicomotor, de fala, audição, dificuldades de alfabetização e aprendizagem, a fim de estabelecer alguns critérios de inclusão na amostra, ou seja, as crianças cujos pais apontaram alguma queixa/ dificuldade significativa nas questões cruciais, abaixo especificadas, foram excluídas da amostra. Os professores também preencheram o Questionário aos Professores com questões de múltipla escolha sobre a aprendizagem, repetência escolar, dificuldades específicas de leitura e/ou escrita, dificuldades de linguagem, fala, audição, atenção e memória, também como auxiliar dos critérios de inclusão da amostra.

As crianças foram avaliadas uma a uma, nas dependências das próprias escolas, em uma sala silenciosa, previamente escolhida para este fim. Para estabelecer os critérios de inclusão, juntamente com a análise dos questionários, foram realizados: Meatoscopia, Triagem do Procesamento Audiométrico e Bateria de Triagem Auditiva (Zaida, 200I).

\section{Resultados e Discussão}

A utilizaçãor do Teste de Shapiro Wilk constatou que a distribuição dos dados não obedeceu à curva normal. Sendo assim, o teste utilizado para verificar a significância estatística foi o Teste não-paramétrico de Kruscal-Wallis, adotando-se como nível de rejeição para hipótese de nulidade o valor de $5 \%$ (os valores onde $\mathrm{p}<0,05$, foram assinalados com *).

As Tabelas de I a 4 apresentam os dados relativos ao Teste de Normalidade de Shapiro Wilk e os valores mínimos, máximos, médios, desvio padrão e $p$-value do Teste Kruscal Wallis relativos à comparação do número de acertos dos indivíduos das faixas etárias de 8,9 e 10 anos nos subtestes.

Observando a Tabela I, pode-se perceber que a diferença no desempenho dos indivíduos de 8, 9 e 10 anos foi estatisticamente significativo, melhorando conforme a idade aumentou. A melhora com relação ao aumento de idade é relatada por vários autores que estudam o comportamento de crianças em testes de Processamento Auditivo (Willeford, 1976, White, 1977; Zaidan, 200I; Costamilan, 2004; Capovilla, 2004). Esse efeito de melhora com o aumento da idade é considerado positivo já que evidencia a capacidade do teste de avaliar a maturação do SNAC. Esses resultados foram similares aos de Zaidan (200I) no subteste Fala Filtrada, que encontrou valores médios de acertos para 8 anos de 34,3; para 9 anos 33,3 e para 10 anos, 36,5, com desvio padrão variando entre 2,2 e 3,5. Além disso, a autora também observou diferença estatisticamente significativa na

Tabela I - Análise do valor de normalidade pela comparação do número de acertos no subteste Fala Filtrada por idade

\begin{tabular}{ccccccc}
\hline $\begin{array}{c}\text { Faixa } \\
\text { Etária }\end{array}$ & $\begin{array}{c}\text { Valor de } \\
\text { normalidade }\end{array}$ & Mínimo & Máximo & Média & DP & $\begin{array}{c}\text { Nível de } \\
\text { significância }(p)\end{array}$ \\
\hline $\begin{array}{c}8 \text { anos } \\
(\mathrm{N}=22)\end{array}$ & $0,0157^{*}$ & 31 & 38 & 35,41 & 1,74 & $0,04^{*}$ \\
9 anos & $0,0324^{*}$ & 32 & 39 & 35,68 & 2,01 & \\
$(\mathrm{~N}=22)$ & & 33 & 39 & 37,08 & 1,93 & \\
10 anos & $0,0467^{*}$ & 33 & & & & \\
$(\mathrm{~N}=13)$ & & & & & &
\end{tabular}

* Valor estatisticamente significativo 
Tabela 2 -Análise do valor de normalidade pela comparação do número de acertos no subteste Fala no Ruído por idade

\begin{tabular}{ccccccc}
\hline $\begin{array}{c}\text { Faixa } \\
\text { Etária }\end{array}$ & $\begin{array}{c}\text { Valor de } \\
\text { normalidade }\end{array}$ & Mínimo & Máximo & Média & DP & $\begin{array}{c}\text { Nível de } \\
\text { significância } \\
(p)\end{array}$ \\
\hline $\begin{array}{c}8 \text { anos } \\
(\mathrm{N}=22)\end{array}$ & 0,3409 & 32 & 39 & 35,36 & 1,87 & 0,15 \\
9 anos & $0,0470^{*}$ & 32 & 40 & 36,41 & 2,21 & \\
$(\mathrm{~N}=22)$ & & 33 & 39 & 36,62 & 1,71 & \\
10 anos & 0,1170 & $33)$ & & & & \\
$(\mathrm{N}=13)$
\end{tabular}

comparação dos indivíduos das faixas etárias de 6 a II anos.

Com relação ao subteste Fala no Ruído, apesar de os resultados melhorarem conforme o aumento da idade, essa diferença não foi estatisticamente significativa, como pode ser observado na Tabela 2. Esses dados corroboram os encontrados por Zaidan (200I). O subteste Fala no Ruído é o único a não demonstrar esse efeito de melhora em sua pesquisa. Semelhantemente aos resultados evidenciados na presente pesquisa, os valores médios de acertos encontrados por Zaidan (200I) foram para os indivíduos de 8 anos a média de acertos de 35; para os indivíduos de 9 anos, 34,2 e para os indivíduos de 10 anos, 36,4 com desvio padrão variando entre I,3 e 2,6.

Esses resultados podem ser explicados pela natureza da tarefa, ou simplesmente por um efeito de amostra. Pesquisas com amostras mais significativas poderiam elucidar de maneira mais clara essas questões.

As diferenças nos desempenhos dos indivíduos de 8, 9 e 10 anos no subteste Palavras Competitivas também não apresentou diferença estatisticamente significativa na análise conjunta das faixas de idade, conforme os dados sumariados na Tabela 3. Apesar de haver melhora nos valores médios de acertos com o aumento da idade, o fato de essas diferenças não serem estatisticamente significativas pode estar relacionado ao grande desvio padrão dos desempenhos intragrupos, principalmente na faixa etária de 10 anos. Esses resultados diferem daqueles encontrados por Zaidan (200I) que considerou esse subteste o mais eficiente para separar os grupos etários.

É importante lembrar que a faixa de idade avaliada por Zaidan (200I) foi bem mais ampla, o que permite

Tabela 3 - Análise do valor de normalidade pela comparação do número de acertos no subteste Palavras Competitivas por idade

\begin{tabular}{|c|c|c|c|c|c|c|}
\hline $\begin{array}{l}\text { Faixa } \\
\text { Etária }\end{array}$ & $\begin{array}{c}\text { Valor de } \\
\text { normalidade }\end{array}$ & Mínimo & Máximo & Média & DP & $\begin{array}{l}\text { Nível de } \\
\text { significância } \\
(p)\end{array}$ \\
\hline $\begin{array}{c}8 \text { anos } \\
(\mathrm{N}=22)\end{array}$ & $0,0083^{*}$ & 77 & 94 & 85,45 & 5,36 & \\
\hline $\begin{array}{c}9 \text { anos } \\
(\mathrm{N}=22)\end{array}$ & 0,2208 & 79 & 93 & 87 & 4,33 & 0,29 \\
\hline $\begin{array}{l}10 \text { anos } \\
(\mathrm{N}=13)\end{array}$ & $0,0296^{*}$ & 79 & 97 & 88,38 & 5,04 & \\
\hline
\end{tabular}

* Valor estatisticamente significativo 
uma observação mais clara dos efeitos do aumento da idade. Além disso, nessa pesquisa não há evidências de comparação entre as faixas etárias duas a duas, não sendo possível comparar a melhora nessas idades especificamente. Com relação aos dados quantitativos, os valores médios de acertos encontrados por Zaidan (200I) foram: para 8 anos de 87,8 , para 9 anos 88,8 e 91,8 para 10 anos, com desvio padrão variando entre 2,5 e 4,9 . subteste Fala Filtrada e no escore total da Bateria. Percebe-se também que o desvio padrão intragrupos foi bastante elevado no subteste Palavras Competitivas e no escore total da Bateria, demonstrando que os resultados tendem a variar bastante dentro da própria faixa etária estudada.

Quando se avaliam habilidades tão interligadas com as funções cognitivas como é o caso das habilidades auditivas, diversos fatores podem interferir nos

Tabela 4 - Análise do valor de normalidade pela comparação do número de acertos no Total da Bateria de Triagem do Processamento Auditivo por idade

\begin{tabular}{ccccccc}
\hline $\begin{array}{c}\text { Faixa } \\
\text { Etária }\end{array}$ & $\begin{array}{c}\text { Valor de } \\
\text { normalidade }\end{array}$ & Mínimo & Máximo & Média & DP & $\begin{array}{c}\text { Nível de } \\
\text { significância } \\
(p)\end{array}$ \\
\hline $\begin{array}{c}8 \text { anos } \\
(\mathrm{N}=22)\end{array}$ & $0,0219^{*}$ & 146 & 167 & 156,23 & 5,62 & \\
9 anos & 0,2497 & 148 & 170 & 159,09 & 5,72 & $0,04^{*}$ \\
$(\mathrm{~N}=22)$ & & 148 & 174 & 162,08 & 7,20 & \\
10 anos & $0,0357^{*}$ & 140 & & & & \\
\hline
\end{tabular}

* Valor estatisticamente significativo

Considerando o Total da Bateria de Triagem do Processamento Auditivo, que é o somatório dos acertos em cada um dos subtestes (Fala Filtrada, Fala no Ruído e Palavras Competitivas), houve diferença estatisticamente significativa na análise conjunta do desempenho dos indivíduos das três faixas etárias. Esses resultados concordam com os de Zaidan (200I). Com relação aos dados quantitativos, é possível perceber que os valores médios de acertos apresentados pelos indivíduos desta pesquisa no Total da Bateria foram ligeiramente maiores que na pesquisa de Zaidan (200I), com exceção da faixa etária de 9 anos.

Ao analisar o comportamento genérico dos indivíduos ao longo de toda a Bateria de Triagem do Processamento Auditivo pôde-se observar uma melhora dos valores médios de acertos apresentados pelos indivíduos conforme a faixa etária aumentou em todos os subtestes e no Total da Bateria. Porém essa diferença só foi estatisticamente significativa no resultados, tornando a variação desses uma característica, de certa maneira, esperada. Musiek e Lamb (1999), destacaram que, apesar de alguns resultados nos testes de Processamento Auditivo em crianças parecerem expressivos, freqüentemente há uma grande variabilidade entre os testes e os indivíduos, o que torna sua interpretação difícil. Segundo os autores, essa variabilidade pode estar relacionada à natureza dos testes, bem como aos problemas auditivos que afetam a criança, além de fatores psicológicos, educacionais, lingüísticos, sociais e maturacionais, que podem estar relacionados à validade dos testes centrais.

É consenso para a comunidade científica pesquisada que qualquer método de avaliação deve passar por uma análise criteriosa de todas as variáveis envolvidas por meio da aplicação em um grande número de indivíduos, a fim de comprovar a validade e a confiabilidade, além de estabelecer limites confiáveis de normalidade. Mesmo que isso ainda não tenha 
ocorrido no caso da Bateria de Triagem do Processamento Auditivo proposta por Zaidan (200I), os resultados preliminares das pesquisas da autora e deste estudo mostraram ser bastante promissores no campo dos estudos Neuroaudiológicos. Pôde-se perceber que a bateria foi elaborada com extremo cuidado, respeitando as características lingüísticas da população avaliada, seguindo critérios internacionais consagrados, além de apresentar ótima qualidade de gravação, o que foi confirmado por Capovilla (2004). A administração do teste é fácil, prática e rápida, não exige equipamentos sofisticados, o que permite a aplicação no próprio ambiente escolar, é de fácil interpretação e representa um desafio motivador para as crianças, que responderam bem aos subtestes. Essas características são compatíveis com várias recomendações no que diz respeito aos objetivos de um processo de triagem (Northen \& Downs, 1989; Bellis \& Burke, 1996; Bess \& Humes, 1998).

Sendo assim, destaca-se a importância de mais pesquisas utilizando a bateria numa amostra mais significativa a fim de avaliar aspectos como diferenças de orelha, de gênero, de faixa etária, de escolaridade e para melhor estudar a capacidade do teste em diferenciar crianças com desenvolvimento normal de crianças com DPA e/ou transtornos de aprendizagem.

\section{Referências}

Alvarez, A. M. M. A., Caetano, A. L., \& Nastas, S. S. (1997). Processamento Auditivo Central: O que é isto? Fono Atual, I, 346-349.

American Speech-Language-Hearing Association - ASHA (1996). Central auditory processing: Current status and implications for clinical practice. American Journal of Audiology, 5, 4I-54.

Baran, J. A., \& Musiek, F. E. (1999). Behavioral assessment of central auditory nervous system. Em F. E. Musiek \&W. F. Rintelmann (Orgs.), Contemporany perspectives in hearing assessment (pp. 375-4I3). Boston,MA: Allyn \& Bacon.

Bellis, T. J. (1996). Assessment and management of central auditory processing disorder in educational setting. San Diego,CA: Singular.
Bellis, T. J., \& Burke, J. R. (1996). Screening. Em T.J. Bellis (org.) Assessment and management of central auditory processing disorders in the educational settings (pp. 9I-II2). San Diego,CA: Singular.

Bess, F. H., \& Humes, L. E. (1998). Fundamentos de Audiologia. Porto Alegre,RS: Artes Médicas.

Capovilla, A. G. S., Capovilla, F. C., \& Silveira, F. B. (2000). Distúrbios de discriminação e memória fonológica em problemas de leitura e escrita. Em M. J. Gonçalves, E. C. Macedo, A. L. Sennyey \& F. C. Capovilla (Orgs.), Tecnologias em (re)habilitação cognitiva - A dinâmica clínicateoria-pesquisa (pp. 236-243). São Paulo, SP: Centro Universitário São Camilo.

Capovilla, F. C. (2004). Processamento Auditivo Central: Demonstrando a Validade de uma Bateria de Triagem para Crianças de 6 a II anos (2004) Em F. C. Capovilla (Org.), Neuropsicologia e aprendizagem: uma abordagem multidisciplinar (pp. II7-|4I). (2 ed.). São Paulo,SP: Memnon.

Chermak, G. D., \& Musiek, F. E. (1992). Managing central auditory processing disorders in children and youth. Journal of the American Academy of Audiology, I (3), 6I-65.

Costa, M. I. D. (2003). Processamento auditivo e compreensão leitora. Dissertação de Mestrado, Pontífice Universidade Católica do Rio Grande do Sul, Porto Alegre.

Costamilan, C. M. (200I). O processamento auditivo central em crianças com queixa de dificuldades de aprendizagem. Monografia, Universidade Federal de Santa Maria, Santa Maria.

Costamilan, C. M. (2004). Processamento auditivo em escolares: estudo longitudinal. Dissertação de Mestrado, Universidade Federal de Santa Maria, Santa Maria.

Damasceno, Y. S. L., \& Russo, I. C. P. (2004). Processamento auditivo em crianças com e sem dificuldades de leitura $\mathrm{e}$ escrita. Em Anais do $19^{\circ}$ Encontro Internacional de Audiologia, Bauru/SP.

Emanuel, D. (2002). The Auditory Processing Battery: Survey of Common Pratices. Journal of the American Academy of Audiology, I3(2), 93-II7.

Flowers, A. (1964). Central auditory abilities of normal and lower group readers. Journal of Otolaryngology, 3(3), 21 I214 . 
Frota, S. (2004). Processamento Auditivo: estudo em crianças com transtornos específicos de leitura e escrita. Em Anais do $19^{\circ}$ Encontro Internacional de Audiologia, Bauru/SP.

Ferre, J. M. (1997). Processing power: a guide to CAPD assessment and management. San Antonio,TX: Comunication Skill Builders.

Katz, J. (1992). Classification of auditory processing disorders. Em J. Katz, N. Stecker, \& D. Henderson (Org.), Central auditory processing: a transdisciplinary view (pp. 8I-9I). St. Louis,MO: Year Book.

Katz, J., \& Wilde, L. (1989). Distúrbios da percepção auditiva em crianças. Em J. Katz (Org.), Tratado de Audiologia Clínica (pp. 674-694). São Paulo: Manole.

Keith, R. W. (1986). SCAN: A Screening Test for Central Auditory Processing Disorders. San Antonio,TX: The Psychological Corporation.

Keith, R. W. (1988). Tests of Central Auditory Function. Em R. Roeser \& M. P. Downs (Orgs.), Auditory disorders in school children. Identification and remediation (pp. 83-97). New York, NY: Thieme Medical.

Keith, R. W., \& Pensak, M. L. (1991). Central Auditory function. Otolaryngologic Clinics of North America, 24, 37I-379.

Musiek, F. E., \& Lamb, L. (1999). Avaliação auditiva central: uma visão geral. Em J. Katz (Org.), Tratado de audiologia clínica (pp. 195-209). (4ª ed.). São Paulo, SP: Manole.
Northen J. L., \& Downs, M. P. (1989). Desenvolvimento do Comportamento Auditivo. Em J. L. Northen \& M. P. Downs (Orgs.), Audição em Crianças (pp. I0I-125). São Paulo,SP: Manole.

Ribas-Guimarães, A. (2000). Uma análise da percepção auditiva em crianças com e sem atraso no desenvolvimento da linguagem. Jornal Brasileiro de Fonoaudiologia, 5, 60-65.

Sanches, M. L., \& Alvarez, A. M. M. A. (2000). Avaliação do PAC em crianças portadoras de transtornos de aprendizagem. Acta AWHO, 19, 185-188.

White, E. (1977). Children's performance on the SSW test and Willeford battery: Interim clinical data. Em R. W. Keith (Org.), Central auditory dysfunction (pp. 319-340). New York, NY: Grune \& Stratton.

Willeford, J. A. (1976). Central auditory function in children with learning disabilities. Audiology Hearing. Education, 2, 12-20.

Zaidan, E. (200I). Desenvolvimento de uma bateria de testes de triagem da função auditiva central em pré-escolares e escolares na faixa etária de 6 a I/ anos. Dissertação de Mestrado, Universidade Federal de São Paulo, São Paulo.

Recebido em: 21/02/2006

Revisado em: 10/05/2006

Aprovado em: 14/06/2006

Sobre as autoras

Larissa Fortunato Simon (larifono@yahoo.com.br) é fonoaudióloga, mestranda em Distúrbios da Comunicação Humana pela Universidade Federal de Santa Maria (UFSM).

Angela Garcia Rossi (angelarossi@terra.com.br) é fonoaudióloga, professora e doutora adjunto do Departamento de Fonoaudiologia da Universidade Federal de Santa Maria (UFSM).

Endereço para correspondência

Larissa Fortunato Simon

Rua Professora Viero, 57I, apto 224

95040-520, Caxias do Sul/RS 


\section{ANEXO I}

\section{PROTOCOLO DA TRIAGEM DO PROCESSAMENTO AUDITIVO DE ZAIDAN (200I)}

FALA FILTRADA: DATA:

FALA RUÍDO: $\quad$ DATA:

\begin{tabular}{|l|l|l|l|l|}
\hline \multicolumn{1}{|c|}{ OD } & \multicolumn{1}{c|}{ OD } & \multicolumn{1}{c|}{ OE } \\
\hline a) chifre & a) vila & & a) banca & a) livre \\
\hline b) queijo & b) time & & b) reta & b) treme \\
\hline 1. bode & 1. suco & & 1. disse & 1. trave \\
\hline 2. milho & 2. dente & FALA FILTRADA & 2. pano & 2. dança \\
\hline 3. nave & 3. pura & OD: & 3. noite & 3. missa \\
\hline 4. fundo & 4. cesta & OE: & 4. gorda & 4. roça \\
\hline 5. lado & 5. grade & & 5. risco & 5. fibra \\
\hline 6. louco & 6. manga & & 6. corpo & 6. gente \\
\hline 7. nada & 7. riso & FALA RUÍDO & 7. nunca & 7. lixo \\
\hline 8. minha & 8. rua & OD: & 8. zebra & 8. soro \\
\hline 9. uva & 9. tira & OE: & 9. circo & 9. manda \\
\hline 10. pires & 10. cinto & & 10. verde & 10. lente \\
\hline 11. sino & 11. dia & & 11. fala & 11. pulga \\
\hline 12. bolsa & 12. testa & & 12. vinho & 12. canta \\
\hline 13. pena & 13. beco & & 13. ilha & 13. ida \\
\hline 14. cinco & 14. sala & & 14. palco & 14. ave \\
\hline 15. padre & 15. creme & & 15. mundo & 15. fita \\
\hline 16. zíper & 16. lata & & 16. justo & 16. bala \\
\hline 17. traço & 17. queda & & 17. sede & 17. zona \\
\hline 18. lindo & 18. tarde & & 18. lousa & 18. muro \\
\hline 19. senta & 19. sara & & 19. gripe & 19. seda \\
\hline 20. drama & 20. pasta & & 20. disco & 20. tiro \\
\hline
\end{tabular}


PALAVRAS COMPETITIVAS:

DATA:

\begin{tabular}{|c|c|c|c|}
\hline OD & OE & OD & OE \\
\hline a) ave & creme & c) caldo & corpo \\
\hline b) fita & dia & d) feira & suco \\
\hline 1. bola & time & 26. pura & calo \\
\hline 2. zero & milho & 27. grito & barco \\
\hline 3. ninho & fundo & 28. nave & noite \\
\hline 4. lente & nunca & 29. senta & banca \\
\hline 5. quente & pulga & 30. chifre & sempre \\
\hline 6. gripe & pista & 31. treme & sente \\
\hline 7. justo & mundo & 32. fina & livre \\
\hline 8. manda & cinto & 33. padre & muita \\
\hline 9. sete & gorro & 34. ele & riso \\
\hline 10. dente & verde & 35. asa & mia \\
\hline 11. canta & santo & 36. gira & vila \\
\hline 12. piso & risco & 37. bico & jipe \\
\hline 13. pasta & nossa & 38. cara & garfo \\
\hline 14. ponta & sombra & 39. mudo & brinco \\
\hline 15. cesta & zebra & 40. bolsa & grupo \\
\hline 16. cinza & zona & 41. fada & marca \\
\hline 17. rica & tarde & 42. pinta & braço \\
\hline 18. trave & pena & 43. lenço & classe \\
\hline 19. vale & sede & 44. tiro & letra \\
\hline 20. bala & isca & 45. dado & surdo \\
\hline 21. uva & lindo & 46. diga & monte \\
\hline 22. disco & pires & 47. ria & disse \\
\hline 23. lia & muro & 48. data & dorme \\
\hline 24. Iouco & salto & 49. tia & nela \\
\hline 25. queijo & circo & 50. pia & seda \\
\hline
\end{tabular}

OD:

OE: 